\title{
Issues in Nonlinear Hyperperfect Numbers
}

\author{
By Daniel Minoli
}

\begin{abstract}
Hyperperfect numbers (HP) are a generalization of perfect numbers and as such share remarkably similar properties. In this note we show, among other things, that if $m=p_{1}^{\alpha_{1}} p_{2} \alpha_{2}$ is 2 -HP then $\alpha_{2}=1$, with $p_{1}=3, p_{2}=3^{\alpha_{1}+1}-2$; this is in agreement with the structure of the perfect case $(1-\mathrm{HP})$ stating that such a number is of the form $m=p_{1}^{\alpha_{1}} p_{2}$ with $p_{1}=2$ and $p_{2}=2^{\alpha_{1}+1}-1$.
\end{abstract}

1. Introduction. Integers having "some type of perfection" have received considerable attention in the past few years. The most well-known cases are: perfect numbers, [1], [12]-[15]; multiperfect numbers, [1] ; quasiperfect numbers, [2] ; almost perfect numbers, [3]-[5]; semiperfect numbers, [16], [17]; and unitary perfect numbers, [11]. The related issue of amicable, unitary amicable, quasiamicable and sociable numbers [8], [10], [11], [9], [6], [7] has also been investigated extensively.

The intent of these variations of the classical definition appears to have been the desire to obtain a set of numbers, of nontrivial cardinality, whose elements have properties resembling those of the perfect case. However, none of the existing definitions generates a rich theory and a solution set having structural character emulating the perfect numbers; either such sets are empty, or their euclidean distance from zero is greater than some very large number, or no particularly unique prime decomposition form for the set elements can be shown to exist.

This is in contrast with the abundance (cardinally speaking) and the crystalized form of the $n$-hyperperfect numbers ( $n$-HP), [18], [19]. These numbers are a natural extension of the perfect case, and as such share remarkably similar properties, as described below.

We begin with a few definitions.

Definition 1. A natural number $m$ is said to be $n$-hyperperfect if for a positive integer $n$,

$$
m=1+n[\sigma(m)-m-1]
$$

For $n=1$, this reduces to the classical case. Table 1 lists all the $n$-HP $(n>1)$ up to $1,500,000$.

Definition 2. $m=p_{1}^{\alpha_{1}} p_{2}^{\alpha_{2}} \cdots p_{j}^{\alpha_{j}}$ is said to be in canonical form if

$$
p_{1}<p_{2}<\cdots<p_{j}
$$

Received March 16, 1979.

AMS (MOS) subject classifications (1970). Primary 10A20, 10A25. 
In the sequel we assume that numbers are always represented in canonical form; also, $p_{i}$ refers invariably to a prime number.

Definition 3. If $m$ is $n$-HP and $m=p_{1}^{\alpha} p_{2}$, we say that $m$ is a linear $n-\mathrm{Hp}$; otherwise, if $m=p_{1}^{\alpha_{1}} p_{2}^{\alpha_{2}}, \alpha_{2}>1$, we say that $m$ is a nonlinear $n$-HP.

From observation of Table 1 it appears that the only hyperperfect numbers are the linear $n$-HP. In this paper we show that, indeed, some nonlinear forms are impossible; a more general theorem which would state that a necessary and sufficient form for a number to be $n$-HP is that it be a linear $n$-HP, remains to be established.

We confine our discussion to the product of two distinct primes, since this is the simplest case to analyze; naturally, the multiprime case must eventually be resolved if the conjecture alluded to above is to be established.

The following basic theorem of linear $n$-HP gives a sufficient form for a hyperperfect number, [19]:

THEOREM 1. $m$ is a linear $n-H P$ if and only if

$$
p_{2}=\frac{n p_{1}^{\alpha_{1}+1}-(n-1) p_{1}-1}{p_{1}^{\alpha_{1}+1}-(n+1) p_{1}^{\alpha_{1}}+n}
$$

2. Theory. We wish to show, among other things, that if $m=p_{1}^{\alpha} p_{2}^{\alpha}$ is $2-\mathrm{HP}$, then $\alpha_{2}=1$.

THEOREM 2. If $m=p_{1}^{\alpha} p_{2}^{\alpha}$ is 2-HP, then $p_{1}=3$.

Proof. We must have

$$
3 m=2 \sigma(m)-1 .
$$

Substituting $m=p_{1}^{\alpha} p_{2}^{\alpha_{2}}$, we obtain

$$
p_{1}^{\alpha_{1}}(2 \Omega-3 \theta)+p_{1}^{\alpha_{1}-1} 2 \Omega+\cdots+p_{1} 2 \Omega+(2 \Omega-1)=0,
$$

where

Notice that

$$
\theta=p_{2}^{\alpha_{2}}, \quad \Omega=p_{2}^{\alpha_{2}}+p_{2}^{\alpha_{2}-1}+\cdots+p_{2}+1
$$

$$
2 \Omega-3 \theta=-p_{2}^{\alpha_{2}}+2\left(\frac{p_{2}^{\alpha_{2}}-1}{p_{2}-1}\right)<-p_{2}^{\alpha_{2}}+\frac{2}{p_{2}-1} p_{2}^{\alpha_{2}}=p_{2}^{\alpha_{2}}\left\{\frac{2}{p_{2}-1}-1\right\} \leqslant 0
$$

if $p_{2} \geqslant 3$; the case $p_{2}=2$ is impossible. [20] shows that if

$$
a_{0} x^{n}+a_{1} x^{n-1}+\cdots+a_{n}=0
$$

then any root $z$ satisfies

$$
\left|z+\frac{a_{1}}{a_{0}}\right| \leqslant 1 \text {, or }|z| \leqslant 1+\max _{2 \leqslant j \leqslant n-1}\left|\frac{a_{j}}{a_{0}}\right| \text {, or }|z| \leqslant\left|\frac{a_{n}}{a_{0}}\right| .
$$


TABLE 1

$n$-HP up to $1,500,000, n \geqslant 2$

n

2

6

3

12

18

18

12

2

30

11

6

2

60

48

19

132

132

10

192

2

31

168

108

66

35

252

18

132

342

366

390

168

348

282

496

540

546

59 m

21

301

325

697

1,333

1,909

2,041

2,133

3,901

10,693

16,513

19,521

24,601

26,977

51, 301

96,361

130,153

159,841

163,201

176,661

214,273

250,321

275,833

296,341

306,181

389,593

486, 877

495,529

542,413

808,861

$1,005,421$

$1,005,649$

$1,055,833$

$1,063,141$

$1,232,053$

$1,284,121$

$1,403,221$

$1,433,701$
Prime decomp

of $m$

$3 \times 7$

$7 \times 43$

$5^{2} \times 13$

$17 \times 41$

$31 \times 43$

$23 \times 83$

$13 \times 157$

$3^{3} \times 79$

$47 \times 83$

$17^{2} \times 37$

$7^{2} \times 337$

$3^{4} \times 241$

$73 \times 337$

$53 \times 509$

$29^{2} \times 61$

$173 \times 557$

$157 \times 829$

$11^{2} \times 1321$

$293 \times 557$

$3^{5} \times 727$

$47^{2} \times 97$

$193 \times 1297$

$133 \times 2441$

$67 \times 4423$

$53^{2} \times 109$

$317 \times 1229$

$79 \times 6163$

$137 \times 3617$

$499 \times 1087$

$463 \times 1747$

$479 \times 2099$

$173 \times 5813$

$401 \times 2633$

$307 \times 3463$

$691 \times 1783$

$829 \times 1549$

$787 \times 1783$

$89^{2} \times 181$ 
This translates into

$\left|p_{1}+\frac{2 \Omega}{2 \Omega-3 \theta}\right| \leqslant 1$, or $\left|p_{1}\right| \leqslant 1+\left|\frac{2 \Omega}{2 \Omega-3 \theta}\right|$, or $\left|p_{1}\right| \leqslant\left|\frac{2 \Omega-1}{2 \Omega-3 \theta}\right|<\left|\frac{2 \Omega}{2 \Omega-3 \theta}\right|$.

Because $2 \Omega-3 \theta<0$, these three bounds may be shown to be equivalent to the bound

$$
p_{1} \leqslant 1+\frac{2 \Omega}{3 \theta-2 \Omega}, \text { or } p_{1} \leqslant 1+\frac{2 p_{2}^{\alpha_{2}}+2 p_{2}^{\alpha_{2}-1}+\cdots+2}{p_{2}^{\alpha_{2}}-2 p_{2}^{\alpha_{2}-1}-\cdots-2}
$$

which, by long division, yields

$$
p_{1} \leqslant 3+\frac{6 p_{2}^{\alpha^{-1}}+6 p_{2}^{\alpha_{2}^{-3}}+\cdots+6}{p_{2}^{\alpha_{2}}-2 p_{2}^{\alpha_{2}-1}-\cdots-2}
$$

or, using the sum formula,

$$
p_{1} \leqslant 3+\frac{6 p_{2}^{\alpha_{2}}-6}{\left(p_{2}-3\right) p_{2}^{\alpha_{2}}+2}=Q\left(p_{2}, \alpha_{2}\right)
$$

Now, $Q\left(p_{2}, \alpha_{2}\right)$ decreases monotonically to 3 as $p_{2} \rightarrow \infty, \alpha_{2}$ fixed; for $p_{2}=3$, $p_{1}=2$ is the only choice; for $p_{2}=5, p_{1}=2$ or 3 ; thus, the maximum value for $Q\left(p_{2}, \alpha_{2}\right)$ must be obtained for $p_{2}=7$; but here $p_{1} \leqslant 4.5$, on the other hand, $Q\left(p_{2}, \alpha_{2}\right)$ increases monotonically to $3+6 /\left(p_{2}-3\right)$ from below as $\alpha_{2} \rightarrow \infty, p_{2}$ fixed. Thus $p_{1} \leqslant 3$. Since $p_{1} \geqslant 3$, [19], we obtain the desired result. Q.E.D.

THEOREM 3. There are no $n$-HP of the form $m=p_{1}^{\alpha} p_{2}^{2}$ with $p_{1}=n+1$; i.e., if an $n$-HP of the form $p_{1}^{\alpha_{1}} p_{2}^{2}$ exists, then $p_{1}>n+1$.

Proof. If $m=p_{1}^{\alpha_{1}} p_{2}^{2}$ is $n$-HP, then $p_{2}$ must satisfy the polynomial

$$
(n+1) p_{1}^{\alpha} p_{2}^{2}=n \frac{p_{1}^{\alpha_{1}+1}-1}{p_{1}-1}\left(p_{2}^{2}+p_{2}+1\right)-n+1
$$

or, solving in $p_{2}$,

$$
p_{2}^{2}\left[p_{1}^{\alpha_{1}}\left(n+1-p_{1}\right)-n\right]+p_{2}\left[n p_{1}^{\alpha_{1}+1}-n\right]+\left[n p_{1}^{\alpha_{1}+1}+p_{1}-n p_{2}-1\right]=0 \text {. }
$$

The discriminant of this quadratic can be shown to be

$$
\begin{aligned}
\Delta= & p_{1}^{2\left(\alpha_{1}+1\right)}\left[n(n+4)-\frac{4 n(n+1)}{p_{1}}\right]+p_{1}^{\alpha_{1}+1}\left[6 n^{2}-8+\frac{4(n+1)}{p_{1}}-4 p_{1}(n-1)\right] \\
& +\left[n(n-4)-p_{1}(4 n)(n-1)\right] .
\end{aligned}
$$

Now if we assume that $p_{1}=n+1$, we get

$$
\begin{aligned}
\Delta & =p_{1}^{2\left(\alpha_{1}+1\right)}\left[n^{2}\right]+p_{1}^{\alpha_{1}+1}\left[2 n^{2}\right]+\left[n^{2}(1-4 n)\right] \\
& =n^{2}\left\{\left(p_{1}^{\alpha_{1}+1}+1\right)^{2}-4 n\right\}=n^{2}\left\{\left[(n+1)^{\alpha_{1}+1}+1\right]^{2}-4 n\right\} .
\end{aligned}
$$


However, $\left[(n+1)^{\alpha_{1}+1}+1\right]^{2}-4 n$ cannot be a perfect square. $\left[(n+1)^{\alpha_{1}+1}+1\right]^{2}$ is a perfect square; the next (smaller) perfect square is $\left[(n+1)^{\alpha_{1}+1}\right]^{2}$; the distance between these two squares is $2(n+1)^{\alpha_{1}+1}+1$; but this is greater than $4 n$ for all $\alpha$ and n. Therefore, $p_{1}>n+1$. Q.E.D.

THEOREM 4. There are no 2-HP of the form $m=p_{1}^{\alpha} p_{2}^{2}$.

Proof. From Theorem 3, if $m=p_{1}^{\alpha} p_{2}^{2}$ is $n$-HP, $p_{1}>n+1$; however, from Theorem 2 a 2 -HP number must have $p_{1}=n+1=3$. This is a contradiction. Thus, no 2-HP of the form $p_{1}^{\alpha} p_{2}^{2}$ exists. Q.E.D.

More importantly,

THEOREM 5. There are no 2-HP of the form $m=p_{1}^{\alpha_{1}} p_{2}^{\alpha_{2}}, \alpha_{2}>1$.

Proof. From Theorem 2, $p_{1}=3$; let $j=\alpha_{1}+1$. $(n+1) m=n \sigma(m)-n+1$ leads to

$$
3^{j} p_{2}^{\alpha_{2}}=\left(3^{j}-1\right)\left(p_{2}^{\alpha_{2}}+p_{2}^{\alpha_{2}-1}+\cdots+1\right)-n+1
$$

for this case.

We thus obtain

$$
P\left(p_{2}\right)=p_{2}^{\alpha_{2}}-\left(3^{j}-1\right) p_{2}^{\alpha_{2}-1}-\left(3^{j}-1\right) p_{2}^{\alpha_{2}-2}-\cdots-3^{j}+2=0 .
$$

Using the same bound used earlier, we obtain $p_{2} \leqslant 3^{j}$; because of the primality condition, $p_{2} \leqslant 3^{j}-1$. For $\alpha_{2}>1$ the following facts hold:

Fact 1. $P\left(3^{j}\right)=2$ since we get a finite telescoping sequence.

Fact 2. $P\left(3^{j}-1\right)<0$ since

$$
P\left(3^{j}-1\right)=-\left(3^{j}-1\right)^{\alpha 1^{-1}}-\left(3^{j}-1\right)^{\alpha_{1}{ }^{-2}}-\cdots-\left(3^{j}-1\right)+1<0 .
$$

Fact 3. $P(x)<0$ for $1<x<3^{j}-1$ since we have

$$
P(x)=x^{\alpha}-\left(3^{j}-1\right) x^{\alpha^{2}-1}-\underbrace{\left(3^{j}-1\right) x^{\alpha_{2}-2}-\cdots-\left(3^{j}-1\right)+1}_{\text {negative since } \alpha_{2}>1}
$$

Therefore,

$$
\begin{aligned}
P(x) & =x^{\alpha_{2}}-\left(3^{j}-1\right) x^{\alpha_{2}-1}-K<x^{\alpha_{2}}-\left(3^{j}-1\right) x^{\alpha_{2}-1} \\
& =x^{\alpha_{2}-1}\left(x-\left(3^{j}-1\right)\right)
\end{aligned}
$$

But $x<\left(3^{j}-1\right)$, therefore, $x-\left(3^{j}-1\right)<0$. This implies $P(x)<0$. This shows that any positive root $r$ of this polynomial satisfies $3^{j}-1<r<3^{j}$, thus no integral values of $p_{2}$ exist.

COROLlaRy 1. If $\alpha_{2}=1, p_{2}=3^{j}-2$ is the only allowable second prime.

Proof. From Theorem 5, if $\alpha_{2}=1, P\left(p_{2}\right)=p_{2}-3^{j}+2=0$ or $p_{2}=3^{j}-2$. Q.E.D. 
For the general $n$-HP case we can obtain a few (weaker) results as follows.

THEOREM 6. If $m=p_{1}^{\alpha} p_{2}^{\alpha}$ is $n$-HP, then

$$
\alpha_{2} \leqslant \frac{\log \left[n\left(p_{1}^{\alpha_{1}}+p_{1}^{\alpha_{1}-1}+\cdots+1\right)+\left(p_{2}-1\right)(n-1)\right]}{\log p_{2}} .
$$

Proof. $(n+1) m=m \sigma(m)-n+1$ leads to

$$
p_{2}^{\alpha_{2}}\left[n\left(p_{1}^{\alpha_{1}}+\cdots+1\right) \frac{p_{2}}{p_{2}-1}-(n+1) p_{1}^{\alpha_{1}}\right]=n\left(p_{1}^{\alpha_{1}}+\cdots+1\right) \frac{1}{p_{2}-1}+n-1
$$

so that

$$
\alpha_{2}=\log \left[\frac{n\left(p_{1}^{\alpha_{1}}+\cdots+1\right)+\left(p_{2}-1\right)(n-1)}{n\left(p_{1}^{\alpha_{1}}+\cdots+1\right) p_{2}-(n+1)\left(p_{2}-1\right) p_{1}^{\alpha_{1}}}\right] / \log p_{2}
$$

from which the result follows. This bound is actually attained in many circumstances. Q.E.D.

THEOREM 7. If $m=p_{1}^{\alpha} p_{2}^{2}$ is $n$ - HP, then $n+2 \leqslant p_{1} \leqslant(n+1)^{2}$.

Proof. As in Theorem 3,

$$
(n+1) p_{1}^{\alpha_{1}} p_{2}^{2}=n \frac{p_{1}^{\alpha_{1}+1}-1}{p_{1}-1}\left(p_{2}^{2}+p_{2}+1\right)-n+1
$$

or, solving in $p_{1}$,

$$
\begin{aligned}
p_{1}^{\alpha_{1}}\left[-p_{2}^{2}+n p_{2}+n\right]+ & p_{1}^{\alpha_{1}-1}\left[n\left(p_{2}^{2}+p_{2}+1\right)\right]+p_{1}^{\alpha_{1}-2}\left[n\left(p_{2}^{2}+p_{2}+1\right)\right] \\
& +\cdots+p_{1}\left[n\left(p_{2}^{2}+p_{2}+1\right)\right]+\left[n p_{2}^{2}+n p_{2}+1_{1}=0 .\right.
\end{aligned}
$$

Note that $-p_{2}^{2}+n p_{2}+n=-p_{2}\left(p_{2}-n\right)+n<0$ since $p_{2}>n$. Using the bounds of Theorem 2, from [20], we obtain

$$
\begin{aligned}
p_{1} & \leqslant 1+\frac{n p_{2}^{2}+n p_{2}+n}{p_{2}^{2}-n p_{2}-n}=n+1+\frac{\left(n+n^{2}\right)\left(p_{2}+1\right)}{p_{2}^{2}-n p_{2}-n} \\
& =n+1+\frac{\left(n+n^{2}\right)\left(p_{2}^{2}-1\right)}{\left[p_{2}-(n+1)\right] p_{2}^{2}+n} .
\end{aligned}
$$

This bound decreases monotonically to $n+1$ as $p_{2} \rightarrow \infty$; thus the maximum is obtained at $p_{2}=n+2\left(p_{2}>p_{1} \geqslant n+1\right)$, so that

$$
p_{1} \leqslant(n+1)+\frac{\left(n+n^{2}\right)(n+3)}{(n+2)^{2}-n(n+2)-n}=(n+1)+\frac{n(n+1)(n+3)}{n+4} \leqslant(n+1)^{2} \text {. }
$$

Note $p_{1} \geqslant n+2$ by Theorem 3 above. Q.E.D. 
3. Conclusion. It appears empirically that the only $n$-HP are the linear $n$-HP. In this paper we have shown that there are no nonlinear 2-HP. A bound on $\alpha_{2}$ for nonlinear $n$-HP, $n \neq 2$, has also been derived. A more general theorem stating that there are not nonlinear $n$-HP (for any $n$ ) is currently being sought.

Computer time (PDP 11/70) for Table 1 was over 10 hours.

Bell Telephone Laboratories

Holmdel, New Jersey 07733

1. L. E. DICKSON, History of the Theory of Numbers, Vol. 1, Chelsea, New York, 1952.

2. H. L. ABBOT ET AL., "Quasiperfect numbers," Acta. Arith., v. 22, 1973, pp. 439-447. MR $47 \# 4915$.

3. M. KISHORE, “Odd almost perfect numbers," Notices Amer. Math. Soc., v. 22, 1975, p. A-380, Abstract \#75FA92.

4. R. P. JERRARD \& N. TEMPERLEY, “Almost perfect numbers," Math. Mag., v. 46, 1973 , pp. $84-87$.

5. J. T. CROSS, “A note on almost perfect numbers," Math. Mag., v. 47, 1974, pp. 230231.

6. P. HAGIS \& G. LORD, “Quasi-amicable numbers,” Math. Comp., v. 31, 1977, pp. 608611.

7. HENRI COHEN, “On amicable and sociable numbers," Math. Comp., v. 24, 1970, pp. 423-429.

8. P. BRATLEY ET AL., "Amicable numbers and their distribution," Math. Comp., v. 24, 1970 , pp. $431-432$.

9. W. E. BECK \& R. M. NAJAR, "More reduced amicable pairs," Fibonacci Quart., v. 15, 1977 , pp. $331-332$.

10. P. HAGIS, "Lower bounds for relatively prime amicable numbers of opposite parity," Math. Comp., v. 24, 1970, pp. 963-968.

11. P. HAGIS, “Unitary amicable numbers," Math. Comp., v. 25, 1971, pp. 915-918.

12. M. KISHORE, "Odd integers $N$ with five distinct prime factors for which $2-10^{-12}<$ $\sigma(N) / N<2+10^{-12}$," Math. Comp., v. 32, 1978, pp. 303-309.

13. L. E. DICKSON, "Finiteness of the odd perfect and primitive abundant numbering with a distinct prime factor," Amer. J. Math., v. 35, 1913, pp. 413-422.

14. P. HAGIS, "A lower bound for the set of odd perfect numbers," Math. Comp., v. 27, 1973, pp. $951-953$.

15. B. TUKERMAN, "A search procedure and lower bound for odd perfect numbers,"

Math. Comp., v. 27, 1973 , pp. 943-949.

16. J. BENKOSKI \& P. ERDÖS, "On weird and pseudo perfect numbers," Math. Comp., v. 28,1974, pp. $617-623$.

17. A. E. ZACHARIOV, "Perfect, semi-perfect and Ore numbers," Bull. Soc. Math. Grèce, v. 13, 1972, pp. 12-22.

18. D. MINOLI \& R. BEAR, "Hyperperfect numbers," Pi Mu Epsilon J., Fall, 1975, pp. $153-157$.

19. D. MINOLI, "Structural issues for hyperperfect numbers," Fibonacci Quart. (To appear.)

20. D. M. YOUNG \& R. T. GREGORY, A Survey of Numerical Analysis, Addison-Wesley, Reading, Mass., 1973. 